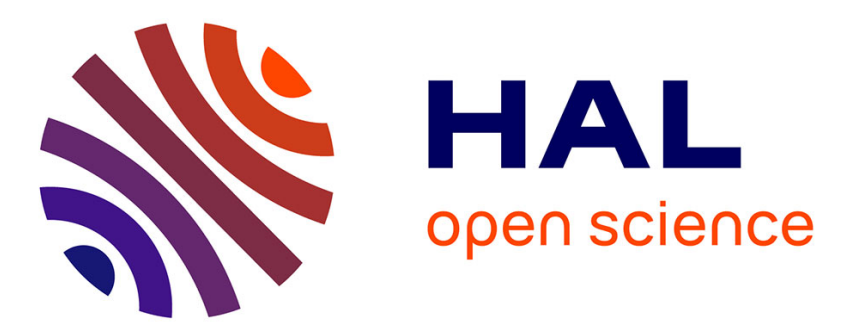

\title{
Inhibition of the alternative lengthening of telomeres pathway by subtelomeric sequences in Saccharomyces cerevisiae
}

Nathalie Grandin, Maria E. Gallego, Charles I. White, Michel Charbonneau

\section{- To cite this version:}

Nathalie Grandin, Maria E. Gallego, Charles I. White, Michel Charbonneau. Inhibition of the alternative lengthening of telomeres pathway by subtelomeric sequences in Saccharomyces cerevisiae. DNA Repair, 2020, 96, pp.102996. 10.1016/j.dnarep.2020.102996 . hal-02985609

\author{
HAL Id: hal-02985609 \\ https://hal.uca.fr/hal-02985609
}

Submitted on 16 Nov 2020

HAL is a multi-disciplinary open access archive for the deposit and dissemination of scientific research documents, whether they are published or not. The documents may come from teaching and research institutions in France or abroad, or from public or private research centers.
L'archive ouverte pluridisciplinaire HAL, est destinée au dépôt et à la diffusion de documents scientifiques de niveau recherche, publiés ou non, émanant des établissements d'enseignement et de recherche français ou étrangers, des laboratoires publics ou privés. 


\title{
Inhibition of the alternative lengthening of telomeres pathway by subtelomeric sequences in Saccharomyces cerevisiae
}

\author{
Nathalie Grandin ${ }^{1}$, Maria Eugenia Gallego ${ }^{1}$, Charles I. White ${ }^{1}$ and \\ Michel Charbonneau ${ }^{1}$ * \\ ${ }^{1}$ GReD Institute, CNRS UMR6293, INSERM U1103, Faculty of Medicine, University \\ Clermont-Auvergne, 28 place Henri Dunant, BP 38, 63001 Clermont-Ferrand Cedex, \\ France
}

* Corresponding author: Michel CHARBONNEAU, GReD Institute, CNRS UMR6293, Faculty of Medicine, University Clermont-Auvergne, 28 place Henri Dunant, 63001 Clermont-Ferrand Cedex, France

e-mail: michel.charbonneau@uca.fr

Running title: Subtelomeric elements inhibit telomere recombination

Keywords: replicative senescence; telomere recombination; subtelomeric Y' elements; Rad52; telomerase-independent telomere maintenance; budding yeast 


\section{Highlights}

* Re-introducing telomerase into a post-senescence type I survivor leads to the generation of a S. cerevisiae strain with $100 \%$ of telomeres containing $Y^{\prime}$ subtelomeric elements.

* The amount of $Y^{\prime}$ subtelomeric elements can influence the selection of the pathway of telomere maintenance by recombination.

* The amount of $Y$ ' subtelomeric elements can influence the response of the cell to telomeric damage caused by telomerase inactivation.

* A S. cerevisiae strain with $100 \%$ of telomeres containing $Y$ ' subtelomeric elements, can grow in the absence of the essential telomere capping protein Cdc13. 


\section{Abstract}

In the budding yeast Saccharomyces cerevisiae, telomerase is constitutively active and is essential for chromosome end protection and illimited proliferation of cell populations. However, upon inactivation of telomerase, alternative mechanims of telomere maintenance allow proliferation of only extremely rare survivors. S. cerevisiae type I and type II survivors differ by the nature of the donor sequences used for repair by homologous recombination of the uncapped terminal $\mathrm{TG}_{1-3}$ telomeric sequences. Type I amplifies the subtelomeric $Y^{\prime}$ sequences and is more efficient than type II, which amplifies the terminal $\mathrm{TG}_{1-3}$ repeats. However, type II survivors grow faster than type I survivors and can easily outgrow them in liquid cultures. The mechanistic interest of studying $S$. cerevisiae telomeric recombination is reinforced by the fact that type II recombination is the equivalent of the alternative lengthening of telomeres (ALT) pathway that is used by $5-15 \%$ of cancer types as an alternative to telomerase reactivation.

In budding yeast, only around half of the 32 telomeres harbor $Y^{\prime}$ subtelomeric elements. We report here that in strains harboring $Y^{\prime}$ elements on all telomeres, type II recombination is inhibited, most likely due to an increase in the efficiency of type I recombination. However, in a temperature-sensitive cdc13-1 mutant grown at semipermissive temperature, the increased amount of telomeric $\mathrm{TG}_{1-3}$ repeats could overcome type II inhibition by the subtelomeric $Y^{\prime}$ sequences. Strikingly, in the $100 \%$ $Y^{\prime}$ strain the replicative senescence crisis normally provoked by inactivation of telomerase, completely disappeared and the severity of the crisis was proportional to the percentage of chromosome-ends lacking $Y^{\prime}$ subtelomeric sequences. The present study highlights the fact that the nature of subtelomeric elements can influence the selection of the pathway of telomere maintenance by recombination, as well as the response of the cell to telomeric damage caused by telomerase inactivation. 


\section{Introduction}

Telomeres are high order assemblies of DNA and proteins located at the ends of linear chromosomes in eukaryotes. The main function of telomeres is to ensure overall genome stability by providing a "cap" that protects against the inappropriate intervention of DNA repair proteins. Chromosome-ends left unprotected following telomere dysfunction become prone to recombination, due to the repetitive nature of their DNA sequences, as well as to end-to-end fusions [1, 2] (de Lange 2009; Maciejowski and de Lange, 2017). During the last three decades, telomeres have become an important field of research and received more and more attention due to the fact that numerous telomere genetic disorders have been described, some of which being implicated in cancer and aging [3-6] (Armanios and Blackburn, 2012; Blackburn et al, 2015; Sarek et al, 2015; Shay and Wright, 2019).

In all dividing cells, telomeres shorten at each cell division due to the endreplication problem that results from incomplete lagging-strand DNA synthesis [7, 8] (Watson, 1972; Soudet et al, 2014), oxidative damage and exonucleolytic processes [9] (Harley et al, 1990). The replication of telomeric DNA by a specialized reverse transcriptase enzyme, telomerase [10, 11] (Vasianovich and Wellinger, 2017; Nguyen et al, 2019), normally compensates for this erosion of telomeres. However, in humans, expression of telomerase is naturally inhibited at the transcriptional level in most somatic tissues. Once their stock of telomeric sequences has been exhausted, cells lacking a telomere maintenance mechanism invoke the DNA damage response (DDR), which results in cell cycle arrest and replicative senescence. This arrest is reversible however, and in cancer cells for instance, events such as activation of oncogenes or mutations in cell-cycle control checkpoint proteins can reverse it. Once these potential cancer cells have resumed cell division, genome instability created by the damage associated with telomere erosion and replicative senescence can lead to either reactivation of telomerase or activation of an alternative lengthening of telomeres (ALT) pathway, principally based on homologous recombination between the repeated telomeric sequences [12-14] (Dilley and Greenberg, 2015; Apte and Cooper, 2017; Sobinoff and Pickett, 2017). Although telomerase has been so far the main target of strategies to disrupt telomere maintenance in cancer [15, 16] (Mocellin et al, 2013; Jafri et al, 2016), ALT-targeting therapeutic approaches have also been developed [17] (Draskovic and Londono- 
Vallejo, 2014). Studies on a mouse model as well as on cultured human cells have established that inhibition of telomerase could lead to utilization of the ALT pathway as a form of resistance [18, 19] (Hu et al, 2012; Queisser et al, 2013). Conversely, targeted inhibition of ALT might provoke telomerase reactivation and, therefore, combined inhibition of both telomere maintenance pathways might achieve full -or at least better- efficiency.

The concepts described above hold true not only for human cancer cells, but for all types of cells experiencing replicative senescence, and in particular have been extensively studied in the yeast Saccharomyces cerevisiae [20] (Teixeira, 2013). Recombination-mediated telomere maintenance was originally discovered in the $S$. cerevisiae est mutants [21] (Lundblad and Blackburn, 1993) and then its equivalent, ALT, in immortalized cancer cells lacking telomerase [22] (Bryan et al, 1995). Yeast est mutants generate either type I survivors, which rely on the Rad51 recombinase for survival through amplification of the subtelomeric $Y$ ' sequences, or type II survivors, which amplify the terminal $\mathrm{TG}_{1-3}$ telomeric repeats in a Rad50-dependent, but Rad51-independent, manner [21, 23-25] (Lundblad and Szostak, 1989; Lundblad and Blackburn, 1993; Le et al, 1999; Teng and Zakian, 1999). Most of the human cell lines that maintain their telomeres by ALT utilize a Rad51-independent mechanism similar to yeast type II survivors [26, 27] (Pickett and Reddel, 2015; Dilley et al, 2016). Based on studies in yeast, this atypical telomere recombination has been hypothesized to often occur by break-induced replication (BIR), a mechanism that can synthesize over $100 \mathrm{~Kb}$ starting from a one-ended double-strand break (DSB) up to the end of the chromosome [13, 28] (Malkova and Ira, 2013; Sobinoff and Pickett, 2017). Yeast ALT-like type II recombination requires the Pol $\delta$ subunit Pol32, while human ALT requires its ortholog PolD3, as well as PolD4, another subunit of Pol $\delta$ [27, 29-31] (Lydeard et al, 2007; 2010; Dilley et al, 2016; Roumelioti et al, 2016). The fact that Pol32, a BIR-implicated actor dispensable for replication and for gene conversion, was required for both type I and type II recombination strongly suggested that BIR functions in both types of telomere maintenance pathways [30] (Lydeard et al, 2007).

Budding yeast cells can survive loss of telomerase by maintaining functional telomeres by recombination through either the classical Rad51-dependent telomeric recombination or the unusual Rad50-dependent, ALT-like, telomeric recombination, 
as explained above. The fact that only around half of the 32 telomeres harbor $Y^{\prime}$ subtelomeric elements might explain the existence of these two types of recombination. Many eukaryotic organisms possess subtelomeric regions that are generally arranged in multiple sequence segments sharing high homology with each other, different from the telomere repeat sequence. In humans, subtelomeres represent a complex patchwork of inter-chromosomal segmental duplications that recently arose during evolution through repeated translocations between chromosome ends [32] (Linardopoulou et al. 2005). Rearrangements or microdeletions in human subtelomeres have been shown to be responsible for mental retardation and malformation syndromes, often caused by haploinsufficiency of subtelomeric genes [33] (De Vries et al. 2003). In the yeast Schizosaccharomyces pombe, subtelomeres, although they are dispensable for mitosis, meiosis and telomere length control, nevertheless play a major role in several aspects of chromosome homeostasis, in particular in telomerase-negative cells, and of gene expression, associated with a buffering capacity that protects the expression of subtelomeric genes from heterochromatin silencing [34] (Tashiro et al. 2017). In S. cerevisiae, about half of the Y' elements contain one of eight YRF1 genes that code for a helicase called Y'-Help1 [35, 36] (Louis and Haber 1992; Yamada et al. 1998). Amplification of $Y$ ' sequences in post-senescence survivors occurs by recombination [21] (Lundblad and Blackburn 1993), but the finding that the expression of helicasecoding Y' elements absent in telomerase-positive wild type cells, increased following telomerase inactivation led to propose that helicase activity might facilitate $Y^{\prime}$ amplification [36] (Yamada et al. 1998).

In the present study, we reasoned that manipulation of the amount of $Y^{\prime}$ sequences might influence the choice of the recombination pathway, I or II, and set out to analyze senescence and post-senescence survival of telomerase-negative $S$. cerevisiae cells with $Y^{\prime}$ subtelomeric elements present on all 32 telomeres. In other words, we asked whether cells having already survived telomerase loss through either type I or type II recombination behaved similarly to naive cells (having never recombined before) when they were further allowed to survive by recombination after having re-acquired telomerase function. The present study reveals that the amount of $Y^{\prime}$ subtelomeric elements can influence the selection of the pathway of telomere 
maintenance by recombination, as well as the response of the cell to telomeric damage caused by telomerase inactivation.

\section{Materials and methods}

\subsection{Yeast strains and media}

Saccharomyces cerevisiae yeast strains used in this study were derivatives of BF264-15Daub (ade1 his2 leu2-3,112 trp1-1a ura3Dns), as described previously [37] (Grandin et al. 1997). Yeast cultures were grown at the indicated temperatures in YEP ( $1 \%$ yeast extract, $2 \%$ bacto-peptone, $0.005 \%$ adenine, $0.005 \%$ uracile) supplemented with $2 \%$ glucose (YEPD) or in selective minimal medium. All strains were made isogenic by back crossing at least five times against our genetic background. Several isolates of $t / c 1 \Delta$, a mutant of the RNA sububit of telomerase, $T L C 1$, namely t/c1::LEU2 and t/c1::TRP1 of either mating type, were used to construct the $100 \% Y^{\prime}$ strains. For the kinetics of senescence, the tlc1s strain bearing TLC1 on a URA3-based plasmid was grown on 5-FOA (5-fluoroorotic acid)containing medium in order to lose the plasmid (counter-selection for URA3), then transferred to YEPD rich medium at $29^{\circ} \mathrm{C}$. The first passage refers to as the first passage after 5-FOA selection. One passage (around 30 population doublings) represents around 2 days in liquid culture and 3 days on agar medium, at $29^{\circ} \mathrm{C}$.

\subsection{Growth competition assays and growth rate measurements}

For growth competition assays, cell cultures from two strains with two different genetic markers and same mating type were mixed together in various proportions and propagated in rich liquid medium (YEPD), the liquid cultures being diluted every 2 days in order for the cells to remain in exponential growth. At each of these passages, cells were withdrawn from the cultures and their growth capacities were determined by the so-called "drop tests" (sometimes referred in the literature to as "spot assays"). To do this, cells from exponential growth cultures were counted with a hematocytometer and the cultures were then serially diluted by $1 / 10^{\text {th }}$, spotted both onto YEPD or selective medium agar plates and incubated at the desired 
temperatures for 2-3 days before being photographed. To evaluate the growth rate of telomerase-negative senescing cells in liquid cultures, cells were counted with a hematocytometer, then diluted (every two days) down to $1 \times 10^{5}$ cells per $\mathrm{ml}$, and the cultures started again at $29^{\circ} \mathrm{C}$. This dilution was chosen because it has been estimated that around 1 in $10^{5} \mathrm{cells} / \mathrm{ml}$ was capable of giving rise to recombining post-senescence survivors [21, 25] (Lundblad and Blackburn; 1993; Teng and Zakian, 1999). Therefore, the $1 \times 10^{5} \mathrm{cells} / \mathrm{ml}$ dilution allows at least one cell to be present in the culture. For all experiments related to kinetics of senescence or competition assays, a minimum of three independent experiments were performed, and a representative one was chosen for illustration.

\subsection{Telomere organization and structure}

To analyze telomere organization and structure, genomic DNAs were hybridized with a 270-bp TG 1 -3 ${ }^{32} \mathrm{P}$-labeled telomeric probe as described previously [37, 38] (Grandin et al, 1997; Grandin and Charbonneau, 2009). About half of S. cerevisiae chromosomes possess subtelomeric $Y^{\prime}$ sequences [39] (Louis and Borts, 1995). Following Xhol digestion, which cuts within these $Y^{\prime}$ regions, telomere tracts of wildtype cells appear as a broad band of $\sim 1.2-1.3 \mathrm{~Kb}$ (comprising $0.9 \mathrm{~Kb}$ from the 3 ' end of the $Y^{\prime}$ element plus $\sim 0.3$ to $0.4 \mathrm{~Kb}$ of terminal $\mathrm{TG}_{1-3}$ tracts), which represents the average length of most chromosomes. In the non-Y' chromosomes, Xhol cutting typically generates fragments migrating at $\sim 2.1,2.3,3.3$, and $3.9 \mathrm{~Kb}$ in Southern blots [25] (Teng and Zakian, 1999). In senescing cells, the disappearance of these non- $Y$ ' fragments attests to the fact that survivors have arisen by homologous recombination. Type II survivors, which amplify the distal $\mathrm{TG}_{1-3}$ telomeric sequences, generate, following Xhol cutting, very long and heterogeneous $\mathrm{TG}_{1-3}$ sequences. In contrast, in type I survivors, which amplify the $Y^{\prime}$ subtelomeric sequences, Xhol cutting reveals a terminal $\mathrm{TG}_{1-3}$ fragment that is consistently $\sim 0.9$ to $1.0 \mathrm{~Kb}$, as well as one or two strong bands above $5 \mathrm{~Kb}$ resulting from the amplification of $\mathrm{Y}^{\prime}$ sequences that are cut by the Xhol site (Fig. 1A). Of note, $Y^{\prime}$ elements are separated by short $\mathrm{TG}_{1-3}$ tracts, allowing type I survivors also to be conveniently detectable with a $\mathrm{TG}_{1-3}$ probe [25] (Teng and Zakian, 1999). Results were analyzed 
using an Amersham ${ }^{\mathrm{TM}}$ Typhoon IP phosphorimager (GE Healthcare) and the ImageQuant TL software.

\subsection{Analysis of transcription of the Y'-short and Y'-long subtelomeric elements}

Total RNA was prepared using the Rneasy kit from Qiagen and $1 \mu \mathrm{g}$ was used for reverse transcription (Promega oligo dT kit). One fifth of the resulting CDNA was used for PCR amplification using the following primers: 5'-Y'-long 5'GAAACAATTAGTGTATTGGATTCG-3' 3' and 5'-long 5' CGAAATCTGCTCCAAAAGATATTC, both amplifying sequences in YFR1-8 YOR396w, and 5'-Y'-short 5'-ATGTTTAATCTAATCAAGGAG-3' and 3'-Y'-short 5'GCATCCAACATGCTGGCCCTT-3', both amplifying sequences in YFL066c. Controls were performed on the constitutively expressed ACT1 gene, using the primers 5'-ACT1-807 5'-GCTGGTTTCTCTCTACCTCAC-3' and 3'-ACT1-1434 5'GAAACACTTGTGGTGAACGAT-3'.

\section{Results}

3.1. Construction of a Saccharomyces cerevisiae strain with homogeneous subtelomeric sequences

Wild-type S. cerevisiae strains contain both $Y^{\prime}$ and non-Y' telomeres and therefore harbor heterogeneous subtelomeres [40] (Horowitz et al. 1984; Fig. 1A, top panel; Supplementary Fig. 1). The existence of these repeated subtelomeric sequences means that $S$. cerevisiae cells having lost telomerase function survive either by using type II recombination between their terminal $\mathrm{TG}_{1-3}$ telomeric sequences or through type I recombination between subtelomeric sequences (Fig. 1A, middle panel). Cells having survived the absence of telomerase though one of these two mechanisms have different growth characteristics and can be recovered separately through culture on agar-based medium or in liquid medium. The majority of postsenescence survivors ( $90 \%$ in our strain background) picked from agar medium are of type I, while in liquid culture, the $\sim 10 \%$ of type II survivors emerging from the initial population of senescing cells (Fig. 1B, right panel), rapidly over-grow type I 
survivors due to their higher rate of growth, ending up representing $100 \%$ of the survivor cell population [25] (Teng and Zakian 1999).

$Y^{\prime}$ subtelomeric elements rapidly spread to all telomeres as type I recombination proceeds, resulting in the percentage of $Y^{\prime}$ telomeres increasing from 50 to $100 \%$ in the post-senescence type I survivors [21, 41] (Lundblad and Blackburn, 1993; Wellinger and Zakian, 2012; Fig. 1A, middle panel). In this work, we have analyzed the consequences of having $100 \% Y^{\prime}$ telomeres $(50 \%$ in the wild type) before loss of telomerase is triggered [40, 41] (Horowitz et al. 1984; Wellinger and Zakian, 2012). To achieve our goal, a type I survivor strain originating from a telomerase-negative strain (t/c1 $\Delta$ ) was transformed with a centromeric plasmid expressing the TLC1 gene (Fig. 1A, middle and bottom panels; Fig. 1B, left panel), which allowed re-acquisition of telomerase-based telomere maintenance (Fig. 1C), in agreement with previous studies [25, 38] (Teng and Zakian 1999; Grandin and Charbonneau, 2009). The "ex-type I" strain, having re-acquired wildtype telomeres after re-introduction of telomerase, will be referred to as "Y' strain" or "Y' ex-type I strain". The behavior of this $Y$ ' strain was compared to that of a $t / c 1 \Delta$ strain expressing $T L C 1$ from a plasmid. This $t / c 1 \triangle p-T L C 1$ control strain has not been exposed to telomerase loss and has wild-type (wt) organization of telomeric and subtelomeric sequences, and will therefore be referred to as "naive strain" (Fig. 1A, top panel; Fig. 1B).

\subsection{Characteristics of the $Y^{\prime}$ strain with $100 \% Y^{\prime}$ telomeres}

The Y' ex-type I strain was induced to undergo replicative senescence by selecting for loss of the plasmid-borne telomerase TLC1 gene on 5-FOA agar medium. A very dramatic observation was that the expected senescence crisis was no longer visible in the 'Y' strain undergoing senescence (Fig. 2). The absence of any sign of senescence in type I survivors exposed to a second round of telomerase loss has previously been observed [42] (Makovets et al. 2008). In addition, we observed that the $Y^{\prime}$ strain could no longer use type II recombination, either when cells were grown on agar medium (Fig. 2A) or in liquid medium (Fig. 2B). This result was quite remarkable, as normally $100 \%$ of the survivors are of type II after propagation in 
liquid culture, as explained above. Interestingly, ex-type II cells (obtained as illustrated in Fig. 1B and C for ex-type I cells), which were extensively grown after reintroduction of telomerase, clearly exhibited senescence crisis upon subsequent loss of telomerase, albeit much less severe than in the wild type (Fig. 2B).

These results suggested either one of two hypotheses. First, the whole population of $Y$ ' strain (ex-type I) survivors did not generate a single type II cell and, therefore, upon liquid expansion, $100 \%$ of the survivors were of type I. Second, the $Y^{\prime}$ survivors were of type I or type II, as usual, but those that were of type I had a faster growth rate than regular type I and type II survivors and, therefore, could not be outgrown during liquid expansion. In this case, $Y$ ' cells would be able to give rise to type II survivors, but those would be outgrown during propagation in liquid culture. To address this issue, we performed growth competition assays. These experiments strongly suggested that survivors from the senescent $Y$ ' strain never generated type II survivors (see Supplementary material and Supplementary Fig. S2).

We next asked whether the fraction of $Y^{\prime}$-containing telomeres was directly correlated with the percentage of inhibition of type II recombination. This was indeed the case, as in successive crosses between $Y^{\prime}$ 'strains and wild-type strains (Fig. 3A), the percentage of type II recombination decreased as the percentage of $Y^{\prime}$ telomeres increased (Fig. 3B). Moreover, the senescence crisis was delayed as the amount of Y' sequences increased and eventually disappeared in the $100 \% Y^{\prime}$ strain (Fig. 3C). An important conclusion of this is that the switch between telomere maintenance by telomerase and that by recombination (and consequently the rate of post-senescence survival) was facilitated by increasing the fraction of $Y^{\prime}$-containing subtelomeric sequences.

The $Y^{\prime}$ subtelomeric elements have been shown to contain motifs characteristic of helicases of the DEAD/DEAH and DEXH subfamilies [35] (Louis and Haber, 1992). The expression of Y'-long and Y'-short elements coding for one of these helicases, the Y'-Help1 helicase, was then found to be induced when telomerase became inactive and this was proposed to promote homologous recombination among Y' elements [36] (Yamada et al. 1998). Here, we found that, contrary to that previous study, the YFR1-8 gene, one of eight YFR1 genes (YRF1-1 to YRF1-8) coding for Y'-Help1, was expressed in wild-type cells and, moreover, its 
level of expression did not vary in the survivors generated following inactivation of telomerase (Supplementary Fig. S3). Therefore, in our strain background at least, the helicase activity produced by the $Y^{\prime}$ elements does not appear to assist in subtelomere amplification during recombination-based survival. In addition, the YFL066c gene containing a Y'-short element coding for one of the Y'-helicase-like proteins [35] (Louis and Haber 1992), was not expressed neither in wild-type cells, nor in telomerase-negative cells (Supplementary Fig. S3).

Importantly, we could demonstrate that the Y' strain, which we see generating only type I survivors, was nevertheless capable of undergoing type II recombination. Prior inactivation of RAD51, essential for type I recombination, resulted in the survivors of the $Y^{\prime}$ strain being of type II (Fig. 4). Therefore, in the absence of Rad51, the $Y^{\prime}$ strain is still capable of performing type II recombination by default.

In summary, our results show that irreversible modifications in telomeric structure generated through type I recombination (in telomerase-positive $Y^{\prime}$ cells) prevent subsequent occurrence of type II recombination if telomerase is again inactivated. On the other hand, under the same conditions, the changes in telomeric structure taking place during type II recombination were reversible and did not prevent cells from subsequently undergoing type I recombination. Thus, when extype II cells that had been transformed with telomerase (which led to re-acquisition of a wild-type profile of non-recombining cells; Fig. 5A), were induced again to undergo senescence following loss of telomerase, they were able to perform type I recombination when cultured on agar medium (Fig. 5B) or type II recombination when cultured in liquid medium (Fig. 5C). Thus, changes in telomeric structure during type II recombination are reversible.

\subsection{Telomerase-negative $Y^{\prime}$ cells can survive longer in the absence of Rad52}

Next, we asked whether Rad52, a recombination mediator, was also (as shown above for Rad51) essential for telomere recombination in the $Y$ ' strain. The first step in homologous recombination is the initiation by nucleolytic degradation (by Mre11Rad50-Xrs2, Sae2, Exo1, Sgs1 and Dna2) of the 5'terminated strands at a DNA double-strand break (DSB) to generate 3'-single-stranded DNA (ssDNA) tails [43] 
(Mimitou and Symington, 2009). After these ssDNA overhangs are bound by RPA, one function of Rad52 is to replace this with the Rad51 recombinase [44] (Symington, 2016). Through the formation of a nucleoprotein filament, Rad51 catalyzes homologous pairing and strand invasion of homologous sequences present elsewhere in the genome, followed by extension of the invading strand by DNA synthesis. Finally, resolution of branched DNA structures takes place, followed by DNA synthesis and ligation of the repaired break [44] (Symington, 2016). Recently, Rad52 was shown to also function earlier, in the attenuation of resection of DSB ends, as well as in regulating the choice between the Exo1 and Sgs1 resection pathways by inhibiting the latter [45] (Yan et al, 2019).

Rad52 is indispensable for the assembly of the Rad51-ssDNA nucleoprotein filament and for the whole homologous recombination process, including that occurring in type I post-senescence survivors, as well as for the Rad50-mediated BIR taking place in type II survivors [46] (Claussin and Chang, 2015). To know whether Rad52 was essential or not for post-senescence survival of $Y^{\prime}$ 'cells, we constructed $t / c 1 \Delta \operatorname{rad} 52 \Delta \mathrm{Y}^{\prime}$ and $t / c 1 \Delta$ rad52 $\Delta$ naive strains as well as their $R A D 52^{+}$ counterparts (Fig. 6). Although at first and second passages, the telomerasenegative $Y^{\prime}$ strain did not seem to require Rad52 for survival (compare patches of cells on right top panels of Fig. 6B (naive) and of Fig. 6C (Y'), it then became evident that later on (at third and fourth passages), the $t / c 1 \Delta$ rad52 $\mathrm{Y}^{\prime}$ cells also died, just like the t/c1 $1 \Delta$ rad52 $\Delta$ naive cells had died earlier. Thus, the type I recombination in the $Y$ ' strain still depends on the canonical pathway of homologous recombination, for which the Rad52 recombination mediator is essential. However the $100 \%$ Y' strain can survive longer than the normal $50 \%$ Y' S. cerevisiae strain in the complete absence of Rad52 function. Since Rad52 regulates the extent of resection of a DSB [45] (Yan et al, 2019), it is possible that the greatly extended survival of the telomerase-negative rad52 $\mathrm{Y}$ ' strain, compared with the wild type, is due to increased amounts of ssDNA that might facilitate the recombination reactions.

Incidentally, we noticed that in both the naive and Y' strains, $R A D 52^{+}$mutants senesced later (not seen on the cell patches because occurring after the second passage) than their rad52 $\Delta$ counterparts (Fig. 6B and C, top panels). This has already been observed by us in senescing cells of different genetic contexts $[38,47]$ 
(Grandin et al. 2001; Grandin and Charbonneau 2009). We interpret this as being the result of early, yet undetectable, Rad52-dependent recombination that repairs the precocious damage induced by the first telomere reaching critically short telomere length.

3.4. Competition between the $T G_{1-3}$ and $Y^{\prime}$ donor sequences following inactivation of the major telomere end protection protein, Cdc13

In a previous study we had shown that the temperature-sensitive cdc13-1 mutation, known to lead to accumulation of ssDNA in telomeric and subtelomeric regions of the chromosomes [48] (Garvik et al, 1995), could itself provoke replicative senescence (in the presence of functional telomerase) when the DNA damage checkpoint had been genetically inactivated [47] (Grandin et al, 2001). The post-senescence survivors of the normal cdc13-1 mec3s strain (50\% Y') obtained on agar medium after a few passages (Fig 7B) were exclusively of type II, as shown also here (Fig. 7C; first two lanes after the wt labeled "50"), as were those of cdc13-1 t/c1 $\Delta$ and cdc13-1 mec3 $t / c 1 \Delta$ strains [47] (Grandin et al, 2001). We took advantage of this to confront two contexts with "opposite" dominant types of recombination events: one in which type I recombination has become the only possible outcome (in the $Y^{\prime} t / c 1 \Delta$ strain) and the other in which the cdc13-1 mutation had established dominance of type II recombination over type I recombination.

To confront these two types of events, we first combined the cdc13-1 and mec3 $3 \Delta$ mutations in strains with different fractions of $Y$ '-containing telomeres in the presence of functional telomerase (Fig. 7A). Successive crosses between cdc13-1 mec3 3 mutants with 50\% $Y^{\prime}$ sequences and 100\% $Y^{\prime}$ strains generated cdc13-1 mec3s mutants with various fractions of $Y$ '-containing telomeres. Following replicative senescence in these $c d c 13-1$ mec3s strains (which was initiated after around 50 population doublings of growth at $29^{\circ} \mathrm{C}$, as previously shown [47] (Grandin et al, 2001), it became evident that when the amount of $Y$ ' sequences attained $94 \%$, two types of post-senescence survivors could be clearly distinguished based on colony size: large colonies produced by fast-growing and small colonies produced by slow-growing survivors (Fig. 7B). Among the slow growing survivors of 
the strains with amounts of $Y$ ' between 75 and 99\% (Fig. 7B, "small colonies"), some did not generate type II recombination (Fig. 7C, lanes 4,5, 8, 9 and 11), unlike the 50\% Y' cdc13-1 mec3 $\Delta$ strain (Fig. 7C, lanes 2 and 3). On the other hand, fast growing cells (Fig. 7B, "big colonies") displayed type II recombination, like the $50 \%$ $Y^{\prime}$ cdc13-1 mec3 $\Delta$ strain (data not shown). In the slow growing survivors of the 7599\% Y' cdc13-1 mec3 $\Delta$ strains that did not generate type II recombination (Fig. 7C, lanes 4,5, 8, 9 and 11), it is not clear whether telomeres are maintained by telomerase or by type I recombination. Based on previous data showing that a cdc13-1 mec3s rad50 $\Delta$ strain did not generate any survivor [47] (Grandin et al, 2001), the $c d c 13-1$ mec3s strain does not seem to be capable of performing type I recombination by default. However, these experiments were done in $50 \%$ Y' strains and it is likely that type I recombination can function in the $75-99 \% Y^{\prime}$ context. Therefore, in these strains, inhibition of type II recombination by the increased amount of ' $Y$ ' sequences might allow non-recombining cells with telomeres maintained by telomerase to remain in the surviving population, at least on agar medium. Dominance of telomerase over type I recombination in these cells would be explained by the relatively small level of telomeric damage induced by $c d c 13-1$ at $29^{\circ} \mathrm{C}$.

In these cdc13-1 mec3s strains, the telomeric damage was moderate since, at $29^{\circ} \mathrm{C}$, cdc13-1 still grows albeit very slowly [48] (Garvik et al, 1995). We next repeated these experiments, but this time in a telomerase-negative (t/c1 $\Delta$ ) background. It has been shown that type II exerts dominance over type I recombination in the cdc13-1 background, as survivors from cdc13-1 t/c1 $\Delta$ strains grown on agar medium were exclusively of type II [47] (Grandin et al, 2001). Following telomerase inactivation at $29^{\circ} \mathrm{C}$, the $99 \% \mathrm{Y}^{\prime}$ cdc13-1 t/c1 $\Delta$ cells underwent type I recombination, meaning that under these circumstances the cdc13-1-induced type II recombination was no longer dominant (Fig. 7D). Furthermore, performing these experiments in several different clones allowed us to observed what appears to be a pattern of recombination intermediate between type I and type II (Fig. 7D).

In summary, increasing the availability of $\mathrm{TG}_{1-3}$ donor sequences by means of the cdc13-1 mutation in cells with telomeric damage can overcome the dominance of the $Y^{\prime}$ donor sequences, at least when the damage is not too severe. However, with 
the maximal damage possible (after telomerase inactivation), cdc13-1-induced recombination on the $\mathrm{TG}_{1-3}$ donor sequences can no longer overcome the dominance of type I recombination on the $\mathrm{Y}^{\prime}$ donor sequences.

3.5. Acquisition of Y' subtelomeric elements by all telomeres allows cells to survive in the absence of essential telomere capping protein, Cdc13

The major telomere end protection protein in $S$. cerevisiae, Cdc13, an essential protein [48] (Garvik et al, 1995), can become dispensable when telomeres are maintained by type II recombination and the DNA damage checkpoint has been genetically inactivated, as attested by the survival of $c d c 13-1$ mec $3 \Delta$ cells at $34-36^{\circ} \mathrm{C}$ [47] (Grandin et al, 2001). This can take place either in the presence or absence of functional telomerase. A full deletion of the $C D C 13$ gene can also be tolerated by the cells in different genetic contexts involving inactivation of telomerase and/or inactivation of the DNA damage checkpoint [49, 50] (Larrivée and Wellinger, 2006; Zubko and Lydall, 2006).

In the current study, $c d c 13 \Delta$ mec3s strains with various amounts of $Y^{\prime}$ sequences, all harboring a centromeric plasmid expressing $C D C 13$, were grown on 5-FOA agar medium to select loss of the CDC13-URA3 construct. The number of clones surviving the absence of $\mathrm{Cdc13}$ was considerably increased in the strain containing $99 \% \mathrm{Y}^{\prime}$ sequences (Fig. 8A). The colonies that appeared in the $50 \%$, $88 \%$ and $94 \% Y^{\prime}$ strains presumably correspond to those in which the plasmid-borne URA3 gene has become mutated, but that still have the plasmid bearing $C D C 13$ and have normal telomeres (Fig. 8A, B). In the $99 \%$ strain, $\mathrm{TG}_{1-3}$ telomeres had apparently completely disappeared, as seen by the absence of the band around 1.2$1.3 \mathrm{~Kb}$, normally representing the average size of the bulk of telomeres (Fig. 8B). It is however possible that the generation of telomeric ssDNA in the absence of Cdc13 might have potentially prevented efficient cutting of the genomic DNA with Xhol, thereby leading to an overestimation of telomere loss. Additional studies will be required to describe in detail the telomere maintenance mechanisms in this strain. Not withstanding this point, we can conclude that the presence of $Y^{\prime}$ subtelomeric 
sequences on all telomeres compensates for loss of telomere end protection by the dedicated protein, Cdc13.

\section{Discussion}

In the present study we have shown that manipulating the subtelomeric sequences of the yeast Saccharomyces cerevisiae directly influences the choice and efficiency of the recombination-based telomere maintenance mechanism, as well as telomere capping and the response to telomeric damage induced by telomerase inactivation. Shedding light on the telomeric processes that control whole genome stability, this approach may also help in developing strategies against cancer, as both telomerase and the telomerase-independent telomere recombination (ALT) pathways, either of which is essential for cancer cell proliferation, have been evolutionary conserved and well studied in yeast.

4.1. In an all-Y'-telomeres strain, cells do not die during the transition between telomerase inactivation and the initiation of telomere recombination

An important clue in trying to understand the overwhelming dominance of type I over type II recombination in ex-type I cells is that the percentage of $Y^{\prime}$ telomeres was inversely proportional to the severity of the senescence crisis (Fig. 3). This is most clearly seen in the $100 \% Y^{\prime}$ strain, which responds to the loss of telomerase by switching to type I telomere maintenance without entering into crisis. These two phenomena are thus linked by a single underlying cause, namely the absence of non-Y' telomeres in these cells. In a previous study, the lack of viability crisis during the "resenescence" of type I survivors was also attributed to the acquisition of $Y$ ' repeats by X-only telomeres [42] (Makovets et al. 2008). Acquisition of $Y^{\prime}$ 'sequences by all telomeres is probably not required for the observed disappearance of crisis. Indeed, deleting, for instance, $Y^{\prime}$ sequences from only one telomere in a 100\% Y' strain would roughly diminish the total amount of $Y^{\prime}$ sequences by $3 \%$. In such a $97 \% Y^{\prime}$ strain, the senescence crisis should not take place, because it is already barely visible when the non- $Y^{\prime}$ telomeres comprise $12 \%$ of chromosome ends $(88 \%$ $Y^{\prime}$ strain of Fig. 3C). 
Senescence crisis corresponds to the period of altered cell growth resulting from progressive telomere erosion. Following crisis, the rate of cell proliferation increases again with the triggering of telomere recombination mechanisms to maintain the uncapped/damaged telomeric DNA substrate. Strikingly, we report here that the $Y^{\prime}$ strains (ex-type I cells) experienced telomere erosion without exhibiting any apparent crisis and ex-type II a delayed and less severe crisis (Fig. 2). Senescence crisis results both from an action of the DNA damage checkpoint on cell cycle progression in response to telomere erosion and from cell death resulting from irreparable chromosome damage. One could imagine that ex-type I cells might generate less damage than type I during telomerase loss-induced telomere erosion. However, this is very unlikely since telomeres have similar sizes and the terminal $\mathrm{TG}_{1-3}$ sequences, which shorten first, did it with similar apparent rates in both types. One could also imagine that activation of the DNA damage checkpoint might be less efficient in the senescing ex-type I cells compared with the senescing type I cells. However, we can think of no plausible reason for this to be the case.

Massive cell death due to irreparable damage subsequent to telomere loss largely contributes to the decline of cell proliferation and takes place independently of the checkpoint (cells die whether or not the checkpoint arrests cell cycle progression). Presumably, chromosome degradation resulting from telomere erosion initiated following telomerase inactivation progresses in a centromere direction and eventually concerns essential genes located centromere-proximal of the $X$ subtelomeric elements (Supplementary Fig. S4). In this respect, an important difference between ex-type I and type I senescing cells, is the presence of very high numbers of copies of the $Y^{\prime}$ elements in the former. We postulate that these represent a barrier that protects from the disappearance of essential genes during telomere erosion and chromosome degradation (Supplementary Fig. S4). By the time telomere erosion attains essential genes, the process of subtelomere amplification has been initiated and allows the cells to survive without any apparent loss of viability.

4.2. High amounts of subtelomeric $Y$ ' sequences allow survival in the absence of the $\mathrm{CDC} 13$ telomere capping protein 
Acquisition of $Y^{\prime}$ subtelomeric sequences by all 32 telomeres allowed cells to survive in the absence of the major, essential telomere capping protein, Cdc13. Once these sequences had spread to all telomeres, just a few population doublings were sufficient to eliminate Cdc13 from the cells, provided cell cycle arrest by the DNA damage checkpoint (triggered by loss of Cdc13) had been previously inactivated by deleting MEC3 (Fig. 8A). In the 100\% Y' cdc13 13 mec3 $\Delta$ strain, the terminal telomeric $\mathrm{TG}_{1-3}$ sequences could no longer be detected on the Southern blots, thereby suggesting that the telomeres had completely disappeared (Fig. 8B). Thus, this strain had presumably lost the capacity to load telomerase on telomere ends (Cdc13 being essential for telomerase loading) and likely continued to maintain functional telomeres owing to type I recombination. Our data on $Y^{\prime}$ amplification confirm a previous study [49] (Larrivee and Wellinger, 2006) and are reminiscent of another situation in which very high amplification of the terminal telomeric $\mathrm{TG}_{1-3}$ sequences provoked by the temperature-sensitive cdc13-1 mutation at semi-permissive temperatures for growth (also with inactivation of the DNA damage checkpoint) similarly allowed survival of the cells in the absence of Cdc13 function when these were further grown at $34-36^{\circ} \mathrm{C}[47,49,50]$ (Grandin et al, 2001; Larrivee and Wellinger, 2006; Zubko and Lydall, 2006).

The apparent loss of telomeres concomitant with amplification of subtelomeric

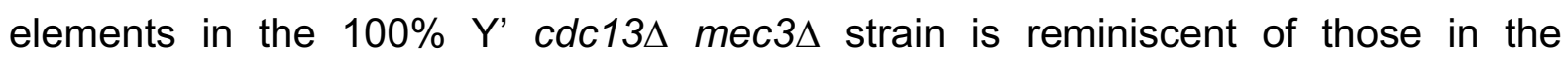
Schizosaccharomyces pombe HAATI strains, survivors of telomerase loss [51] (Jain et al, 2010). These $S$. pombe HAATI survivors still needed capping by Pot1, which also assumes a capping function in telomerase-positive cells [51] (Jain et al, 2010). S. pombe Pot1 has long been regarded as a functional homolog of $S$. cerevisae Cdc13, based on similar effects of their respective mutations. It is clear that an alternative capping complex is present in the $100 \% Y^{\prime}$ survivors, because these cells could survive in the absence of Cdc13. Yet, we note that the $100 \% \mathrm{Y}^{\prime}$ cdc13 13 mec3 3 cells grew very poorly (data not shown), thus suggesting that Cdc13, although not essential, does indeed largely contribute to telomere capping in the $100 \%$ Y' strains.

The fact that both S. cerevisiae 100\% Y' cdc13 13 mec3 3 and S. pombe HAATI cells rely on amplification of subtelomeric elements (and of rDNA for $S$. pombe HAATI [51] (Jain et al, 2010)) for survival is striking. It would be interesting to know 
whether human cancer cells can also amplify subtelomeric sequences to survive, as an alternative to telomerase and ALT. An important issue will also be to uncover the nature of the alternative chromosome capping complex functioning in the $100 \% \mathrm{Y}^{\prime}$

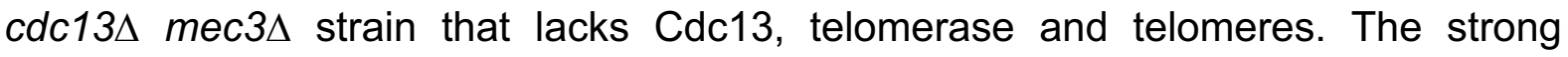
amplification of $Y$ ' sequences, which protects senescing cells from any apparent damage and loss of viability, as discussed above (Supplementary Fig. S3), might counteract progressive erosion of the telomeres due to the end replication problem. However, protection against subtelomere-subtelomere fusion, other possible rearrangements and against degradation by exonucleases must also be taken over by the telomere machinery. Although it is possible that the recombination in the $100 \% Y^{\prime}$ strain is restricted to "productive" $Y$ '-Y' recombination and thus efficiently limits chromosome rearrangements, it is equally possible that protection against all these threats necessitates the intervention of true capping proteins, which could be telomerase, the EST or KU proteins or still unidentified proteins. However, as noted above, when present, Cdc13 plays a major role in telomere end protection in the $100 \%$ Y' strains.

Noticeably, the existence in $S$. cerevisiae of telomere maintenance mechanisms governed by $\mathrm{Cdc} 13$ and telomerase might be more beneficial to the cell than the mechanisms engendered by $Y$ ' spreading and amplification. This alternative $Y$ '-capping mechanism might be harmful to the cells. Indeed, under such conditions, life without the Cdc13-capping complex was possible (albeit only in the absence of the DNA damage checkpoint), but provoked side effects, such as increased sensitivity to DNA-damaging agents [49] (Larrivee and Wellinger, 2006). This may also lead to loss of important extra-telomeric functions of $\mathrm{Cdc13}$, such as the control of interactions between the transcription and replication machineries [52] (Calvo et al, 2019) or the sequestration of unrepaired DSBs at the nuclear envelope [53] (Lisby et al, 2010).

4.3. In an all-Y'-telomeres strain, increasing type I recombination efficiency prevents the occurrence of type II recombination

The dominance of type I over type II recombination in the 100\% Y' strains was overwhelming. Type I recombination in these cells still relied on the Rad51 recombinase and Rad52 mediator and type II recombination was still possible when 
RAD51, essential for type I but dispensable for type II, had been deleted (Fig. 4). Indeed, we have established in parallel that ex-type II survivors, although they grew less well than ex-type I survivors during the senescence period preceding the acquisition of recombination, did grow better than the ex-type I survivors after the senescence crisis (Fig. S2). Thus, according to our protocols, if there were only one type II survivor present among 100,000 survivors of type I in a liquid culture of extype I cells, it would be able to take over the culture. Understanding how telomere dysfunction at the time of replicative senescence crisis can influence cell proliferation once a telomere maintenance mechanism (telomerase or ALT) has been reactivated is of real importance. For instance, it has been observed that telomere dysfunction played a critical role in cancer initiation and progression in a mouse model of prostate cancer, leading to the selection of genomic events favorable to proliferation of cancer cells upon subsequent reactivation of telomerase [54] (Ding et al, 2012).

The homogenization and amplification of the subtelomeric $Y^{\prime}$ sequences throughout the genome of the $S$. cerevisiae strain described here, in addition to dispensing with the Cdc13-capping protein, permitted a very efficient transition between telomere maintenance by telomerase and that by telomere recombination. One might therefore wonder why evolution of the $S$. cerevisiae genome did not generalize $Y^{\prime}$ spreading on all telomeres, particularly given the presence of telomeric $\mathrm{TG}_{1-3}$ repeats on both $X$-only and $X-Y^{\prime}$ telomeres would have facilitated $Y^{\prime}$ spreading. Therefore, there might be an active mechanism used to prevent spreading of $Y^{\prime}$ sequences on all telomeres. We speculate that too strong a $Y^{\prime}$ amplification would represent a threat to genome stability and thus impose a growth penalty (see Fig. 2B). High numbers of subtelomeric repeated sequences might also represent a challenge for the DNA replication machinery and become a substrate for inappropriate homologous recombination. Although they are not as repeated as the telomeric $\mathrm{TG}_{1-3}$ sequences, such highly amplified $\mathrm{Y}^{\prime}$ subtelomeric sequences are probably very difficult to replicate because the distances to replicate without active replication origins would be too large to be replicated without any problem. Indeed, although both $X$ and $Y^{\prime}$ 'subtelomeric elements contain replication origins [55] (Louis 1995), these, like all telomere origins, are late origins very infrequently used by the replication machinery in normal conditions. In support for the assumption that $Y^{\prime}$ amplification is limited by active mechanisms, we observed that when type I 
survivors were grown for a very long time, the amount of $Y^{\prime}$ elements seemed to stabilize, thus suggesting that a mechanism for limiting the amplification of these elements might exist (Supplementary Fig. S5).

\section{Funding}

This work was suppported by a grant from the LIGUE Nationale contre le Cancer (Coordination Recherche Régional Auvergne-Rhône-Alpes et Saône-et-Loire; Comités Puy-de-Dôme et Allier) to MC and NG.

\section{CRediT authorship contribution statement}

Nathalie Grandin: Conceptualization, Methodology, Validation, Formal analysis, Investigation, Data Curation, Writing - Original Draft, Writing - Review \& Editing. Maria Eugenia Gallego: Validation, Formal analysis, Writing - Review \& Editing. Charles I. White: Validation, Formal analysis, Resources, Writing - Review \& Editing. Michel Charbonneau: Validation, Formal analysis, Resources, Data Curation, Writing - Original Draft, Writing - Review \& Editing, Funding acquisition.

\section{Declaration of Competing Interests}

Authors declare no conflict of interest

\section{Acknowledgements}

The authors thank the LIGUE Nationale contre le Cancer for their financial support.

\section{Appendix A. Supplementary data}

Supplemental material related to this article can be found in the online version. 


\section{Figure legends}

Fig. 1. Construction of an S. cerevisiae strain with 100\% Y' subtelomeric sequences. (A) Top: In a wild-type, non recombining, strain (here a telomerase disruptant, t/c1::LEU2, assuming normal telomerase functions owing to the presence of the telomerase RNA, TLC1, on a plasmid, referred to as "naive" because it has never recombined before), all the telomeres contain $X$ subtelomeric elements (orange), but only half contain $Y^{\prime}$ subtelomeric elements (green; see Supplementay Fig. 1 for composition of all 16 chromosomes in subtelomeric elements). The positions of Xhol sites are shown as red dots. When $Y^{\prime}$ sequences are present, the Xhol site closest to telomere end is located inside the $Y^{\prime}$ locus. Cutting genomic DNA with Xhol generates the typical band pattern of telomeres observed by Southern (see below in (B). Zigzag arrows labeled "I "and "II" schematically represent the donor sequences that are used during telomeric recombination of type I and type II, respectively, once telomerase has been inactivated (see middle panel, below). Middle: Telomeric recombination, which is triggered following loss of telomerase function (transfer to 5FOA agar medium to provoke extrusion of the TLC1-URA3 plasmid by counterselecting for URA3 expression), amplifies the telomeric $\mathrm{TG}_{1-3}$ sequences at sites indicated in top panel above. Note the basic differences between the two types of recombination: type II amplifies the terminal telomeric $\mathrm{TG}_{1-3}$ sequences, ending up with long heterogenous terminal $\mathrm{TG}_{1-3}$ sequences, while type I amplifies the subtelomeric ( $Y^{\prime}$ and intercalated $\mathrm{TG}_{1-3}$ ) sequences and are characterized by short terminal telomeric $\mathrm{TG}_{1-3}$ sequences (see panel $\mathrm{B}$, below) and spreading of $\mathrm{Y}^{\prime}$ sequences on all telomeres. Bottom: Reintroduction of telomerase (TLC1) in a type I strain leads to the restoration of a so-called $Y^{\prime}$ wild type strain maintaining functional telomeres by telomerase-dependent mechanisms, but with $100 \%$ Y'containing telomeric sequences instead of $50 \%$ in the wild type (top). (B) Left panel: Representative Southern blot showing telomere organization in a t/c1::LEU2 p-TLC1 strain (lane 1), in a true wild type strain (wt, lane 2) and in a t/c1::LEU2 strain having initiated replicative senescence and the recombination process following loss of the TLC1-URA3 plasmid during growth on 5-FOA agar medium (lane 3). Right panel: Survival from telomerase inactivation (triggered by growth on 5-FOA agar medium; left panel, lane 3 ) is permitted by either one of two types of post-senescence telomeric recombination, as explained above. Numbers at the bottom of the lanes 
indicate the number of passages (around 30 population doublings at $29^{\circ} \mathrm{C}$ per passage; see Materials and methods) following loss of telomerase function. Note that concerning type I kinetics, the two bands located above $5 \mathrm{~Kb}$ that progressively accumulated the radioactive signal correspond to the $Y$ ' subtelomeric elements that are labeled by the $T_{G_{1-3}}$ probe due to the presence of intercalated $T_{G_{1-3}}$ sequences within these $Y$ ' elements. Xhol digestion, ${ }^{32} \mathrm{P}-$-labeled $\mathrm{TG}_{1-3}$ probe for both panels. (C) A post-senescence survivor of type I (t/c1::LEU2 I, lane 2) was transformed with telomerase RNA ( + p-TLC1 URA3) for the indicated number of population doublings (PD). This led to the restoration of telomeres with wild-type characteristics, the size of the bulk of telomeres (about 1.2-1.3 Kb) being identical to that in the wild type (wt, lane 1). Note the increased amount of $Y$ ' sequences (identified as the two strong bands above $5 \mathrm{~Kb}$ as explained above, panel $\mathrm{B}$, right). The strain illustrated in the last lane of these kinetics is typical of what we refer to as a $Y^{\prime}$ or $Y^{\prime}$ ex-type I strain. Xhol digestion, ${ }^{32} \mathrm{P}$-labeled $\mathrm{TG}_{1-3}$ probe.

Fig. 2. Recombination in the $Y$ ' strain, which is of a classical type $I$, is dominant over type II recombination and allows to overcome the usual senescence crisis. (A) Top panel: Telomerase inactivation was triggered by two successive restreaks on 5-FOA agar medium. Numbers of passages as indicated (around 30 population doublings at each passage). Bottom panel: Southern blot performed on genomic DNA from 6 colonies of the $Y$ ' strain above. All examined colonies exhibited a typical profile of type I. Also note the expected strong amplification of $Y$ ' sequences in the bands above $5 \mathrm{~Kb}$ (Xhol digestion, ${ }^{32} \mathrm{P}$-labeled $\mathrm{TG}_{1-3}$ probe). In a total of 60 colonies, none exhibited a type II recombination profile (data not shown), while around 6 out of 60 would be expected in a normal post-senescence wild type strain. (B) Top and middle panels: Growth characteristics of senescing cells of the indicated type in liquid medium following inactivation of telomerase. Bottom panel: Southern blot of the Y' ex-type I strain above as indicated. Type II recombination was never used by this strain, as verified also in around 100 distinct clones from several different $Y^{\prime}$ strains (not shown). Note the very strong amplification of the $Y$ ' sequences visible as two intensely labeled bands above $5 \mathrm{~Kb}$ (Xhol digestion, ${ }^{32} \mathrm{P}_{\text {-labeled }} \mathrm{TG}_{1-3}$ probe). The Southern blot of the ex-type II strain during the senescence kinetics is shown in Fig. 5C. 
Fig. 3. Inhibition of type II recombination increases as $Y^{\prime}$ sequences spread on telomeres. (A) Successive crosses between a normal wild type (50\% Y') strains and $100 \% Y^{\prime}$ strains (also t/C1A p-TLC1 harboring TLC1 on a CEN-URA3 plasmid generated as explained in Fig. 1) generated strains with various amounts of subtelomeric $Y$ ' sequences. (B) These strains were grown and genomic DNA prepared for telomere structure analysis by Southern, either immediately after sporulation of the diploids and loss of the telomerase TLC1 plasmid on 5-FOA (lanes identified "NR" for non recombining) or after 120 population doublings in liquid medium (lanes identified "R" for recombinants). Upon telomerase inactivation, type II recombination is developed in the two $50 \% \mathrm{Y}^{\prime}$ strains, but becomes rarer and rarer as the percentage of ' $Y^{\prime}$ sequences increases and is replaced by type I recombination in the 88 and $100 \% Y^{\prime}$ strains (Xhol digestion, ${ }^{32} \mathrm{P}_{\text {-labeled }} \mathrm{TG}_{1-3}$ probe). Due to the time needed for plasmid loss and sample preparation, telomeres of the non recombining ("NR") $t / c 1 \Delta$ strains have started to shorten (compare with the wild type, lane 1). (C) Drop tests, performed at the indicated numbers of passages, of liquid cultures of the $Y^{\prime}$ 'strains illustrated above, in $B$.

Fig. 4. Type I recombination in the $Y$ ' strain still depends on the canonical pathway of homologous recombination, for which the Rad51 recombinase is essential. A Y' diploid homozygous for $t / c 1 \Delta$ and heterozygous for rad51 (telomerase) on a plasmid, was sporulated after telomerase was inactivated following growth on 5-FOA agar medium. The resulting $Y^{\prime}$ t/c1 $1 \Delta$ rad51 $1 \Delta$ postsenescence survivors, which are normally exclusively of type I (see main text), were this time only of type II, as observed in 7 different clones (Xhol digestion, ${ }^{32} \mathrm{P}$-labeled $\mathrm{TG}_{1-3}$ probe).

Fig. 5. Telomerase-positive cells having previously undergone type II telomeric recombination (strain $t / c 1 \Delta$ ex-type II p-TLC1, panel A), extensively grown following re-introduction of $T L C 1$ telomerase, are capable of subsequently undergoing either type I recombination when TLC1 telomerase plasmid is lost on 5-FOA agar medium and cells grown on agar medium for the indicated numbers of population doublings (PD) (panels B) or type II recombination when, following loss of telomerase on 5FOA agar medium, cells are grown in liquid medium for the indicated numbers of 
passages (one passage every two days, representing around 30 population doublings at $29^{\circ} \mathrm{C}$ ) (panel C). Xhol digestion, ${ }^{32} \mathrm{P}$-labeled $\mathrm{TG}_{1-3}$ probe for all panels.

Fig. 6. Type $I$ recombination in the $Y^{\prime}$ strain still depends on the Rad52 recombination mediator. (A) A $Y^{\prime}$ and a wild type (wt) diploids homozygous for t/c1 $\Delta$ and heterozygous for rad52 $\Delta$ harboring TLC1 (telomerase) on a plasmid were sporulated and in the resulting haploid cells, telomerase was inactivated following growth on 5-FOA agar medium. Growth of these telomerase-negative wt (B, top panel) and $Y^{\prime}$ (C, top panel) strains was analyzed following growth on agar medium for the indicated numbers of passages. As expected, the time of senescence crisis and maximal death in the wild type took place after around 80 population doublings (2 passages) following inactivation of telomerase, but only after around 160 population doublings $\left(4^{\text {th }}\right.$ passage) in the $Y^{\prime}$ strain. Southern teloblots (Xhol digestion, ${ }^{32} \mathrm{P}_{\text {-labeled }} \mathrm{TG}_{1-3}$ probe) performed in parallel indicated that for both the wild type (B, bottom panel) and $Y^{\prime}$ (C, bottom panel) rad52 $\Delta$ strains, there was a progressive decrease in the amplification of the $Y^{\prime}$ sequences as well as a progressive disappearance of all telomeric sequences, occurring simultaneously with death.

Fig. 7. Competition between recombination of type $\mathrm{I}$ induced by increased $\mathrm{Y}^{\prime}$ sequences and recombination of type II induced by inactivation of the major telomere end protection protein, Cdc13. (A) Successive crosses between cdc13-1 mec3A mutants with $50 \% \mathrm{Y}^{\prime}$ sequences and $100 \%$ Y' strains (otherwise wild type for $C D C 13$ and MEC3) generated cdc13-1 mec3 3 mutants with various amounts of subtelomeric $Y$ ' sequences. (B) After sporulation of the diploids, the haploids were passaged three times on agar medium (around 90 population doublings) at $29^{\circ} \mathrm{C}$ to trigger replicative senescence (in the presence of functional telomerase). Two populations of survivors were present in the $75-99 \%$ strains, best seen in the 94\% $Y^{\prime}$ cdc13-1 mec3 $\Delta$ strain

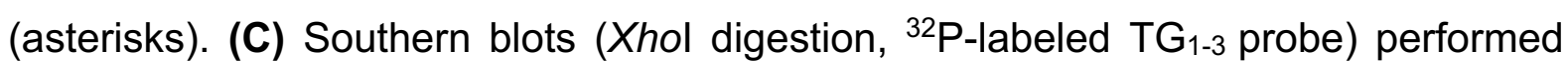
on the smallest visible colonies revealed that some of these could no longer generate type II recombination (see lanes 4, 5, 8, 9 and 11), unlike the $50 \% \mathrm{Y}^{\prime}$

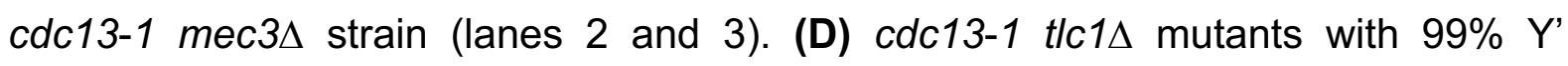
sequences were selected from successive crosses between 100\% $Y^{\prime}$ strains (also 
t/C1S p-TLC1 harboring TLC1 on a CEN-URA3 plasmid, generated as explained in Fig. 1) and cdc13-1 mutants with normal 50\% Y'. Haploids were grown on 5-FOA agar medium at $24^{\circ} \mathrm{C}$ to trigger telomerase inactivation. At this temperature, cdc13-1 generates only small amounts of damage (ssDNA) (loss of telomerase function at temperatures higher than $24^{\circ} \mathrm{C}$ is lethal in cdc13-1). Southern blots (Xhol digestion, ${ }^{32} \mathrm{P}$-labeled $\mathrm{TG}_{1-3}$ probe) performed on 21 different clones after around 120 population doublings evidenced the fact that this time the typical pattern of cdc13-1induced type II recombination had been inhibited by the presence of $Y$ ' sequences on all chromosomes.

Fig. 8. The essential telomere capping protein Cdc13 becomes dispensable when all telomeres have acquired ' $Y^{\prime}$ subtelomeric sequences. (A) Successive crosses between cdc13 13 mec3 $3 \Delta$ mutants (with 50\% Y' sequences) harboring CDC13 on a CEN-URA3 plasmid and 100\% Y' strains (t/c1D p-TLC1 harboring TLC1 on a CENURA3 plasmid otherwise wild type for CDC13 and MEC3) allowed to generate cdc13 13 mec3 $\mathrm{p}-U R A 3-C D C 13$ mutants (note that we selected $T L C 1^{+}$cells at each of the successive crosses) with various amounts of subtelomeric $Y$ ' sequences. Following growth on 5-FOA agar medium, only the cdc13 1 mec3 $\Delta$ strain with $99 \% \mathrm{Y}^{\prime}$ sequences was found to be able to extrude the p-URA3-CDC13 plasmid. (B) All eight $99 \%$ cdc13 13 mec3 $\Delta$ clones analyzed by Southern (Xhol digestion, ${ }^{32}$ P-labeled $\mathrm{TG}_{1-3}$ probe), performed around 120 population doublings following their appearance on 5-FOA agar medium, suggested a total disappearance of the terminal telomeric $\mathrm{TG}_{1-3}$ sequences, which normally appear as a band of around 1.2-1.3 Kb representing the average size of the bulk of telomeres. The first two first lanes show the normal telomeric profiles of URA3-mutated cononies that appeared on 5-FOA in two independent $50 \%$ colonies. 


\section{References}

[1] T. de Lange, How telomeres solve the end-protection problem, Science, 326 (2009) 948-952.

[2] J. Maciejowski, T. de Lange, Telomeres in cancer: tumour suppression and genome instability, Nat Rev Mol Cell Biol, 18 (2017) 175-186.

[3] J.W. Shay, W.E. Wright, Telomeres and telomerase: three decades of progress, Nat Rev Genet, 20 (2019) 299-309.

[4] M. Armanios, E.H. Blackburn, The telomere syndromes, Nat Rev Genet, 13 (2012) 693-704.

[5] E.H. Blackburn, E.S. Epel, J. Lin, Human telomere biology: A contributory and interactive factor in aging, disease risks, and protection, Science, 350 (2015) 1193-1198.

[6] G. Sarek, P. Marzec, P. Margalef, S.J. Boulton, Molecular basis of telomere dysfunction in human genetic diseases, Nat Struct Mol Biol, 22 (2015) 867-874.

[7] J.D. Watson, Origin of concatemeric T7 DNA, Nat New Biol, 239 (1972) 197-201.

[8] J. Soudet, P. Jolivet, M.T. Teixeira, Elucidation of the DNA end-replication problem in Saccharomyces cerevisiae, Mol Cell, 53 (2014) 954-964.

[9] C.B. Harley, A.B. Futcher, C.W. Greider, Telomeres shorten during ageing of human fibroblasts, Nature, 345 (1990) 458-460.

[10] T.H.D. Nguyen, K. Collins, E. Nogales, Telomerase structures and regulation: shedding light on the chromosome end, Curr Opin Struct Biol, 55 (2019) 185-193.

[11] Y. Vasianovich, R.J. Wellinger, Life and Death of Yeast Telomerase RNA, J Mol Biol, 429 (2017) 3242-3254.

[12] M.S. Apte, J.P. Cooper, Life and cancer without telomerase: ALT and other strategies for making sure ends (don't) meet, Crit Rev Biochem Mol Biol, 52 (2017) 57-73.

[13] A.P. Sobinoff, H.A. Pickett, Alternative Lengthening of Telomeres: DNA Repair Pathways Converge, Trends Genet, 33 (2017) 921-932.

[14] R.L. Dilley, R.A. Greenberg, ALTernative Telomere Maintenance and Cancer, Trends Cancer, 1 (2015) 145-156.

[15] S. Mocellin, K.A. Pooley, D. Nitti, Telomerase and the search for the end of cancer, Trends Mol Med, 19 (2013) 125-133.

[16] M.A. Jafri, S.A. Ansari, M.H. Alqahtani, J.W. Shay, Roles of telomeres and telomerase in cancer, and advances in telomerase-targeted therapies, Genome Med, 8 (2016) 69.

[17] I. Draskovic, A. Londono-Vallejo, Telomere recombination and the ALT pathway: a therapeutic perspective for cancer, Curr Pharm Des, 20 (2014) 6466-6471.

[18] J. Hu, S.S. Hwang, M. Liesa, B. Gan, E. Sahin, M. Jaskelioff, Z. Ding, H. Ying, A.T. Boutin, H. Zhang, S. Johnson, E. Ivanova, M. Kost-Alimova, A. Protopopov, Y.A. Wang, O.S. Shirihai, L. Chin, R.A. DePinho, Antitelomerase therapy provokes ALT and mitochondrial adaptive mechanisms in cancer, Cell, 148 (2012) 651-663.

[19] A. Queisser, S. Heeg, M. Thaler, A. von Werder, O.G. Opitz, Inhibition of telomerase induces alternative lengthening of telomeres during human esophageal carcinogenesis, Cancer Genet, 206 (2013) 374-386.

[20] M.T. Teixeira, Saccharomyces cerevisiae as a Model to Study Replicative Senescence Triggered by Telomere Shortening, Front Oncol, 3 (2013) 101.

[21] V. Lundblad, E.H. Blackburn, An alternative pathway for yeast telomere maintenance rescues est1- senescence, Cell, 73 (1993) 347-360.

[22] T.M. Bryan, A. Englezou, J. Gupta, S. Bacchetti, R.R. Reddel, Telomere elongation in immortal human cells without detectable telomerase activity, EMBO J, 14 (1995) 4240-4248.

[23] S. Le, J.K. Moore, J.E. Haber, C.W. Greider, RAD50 and RAD51 define two pathways that collaborate to maintain telomeres in the absence of telomerase, Genetics, 152 (1999) 143-152.

[24] V. Lundblad, J.W. Szostak, A mutant with a defect in telomere elongation leads to senescence in yeast, Cell, 57 (1989) 633-643.

[25] S.C. Teng, V.A. Zakian, Telomere-telomere recombination is an efficient bypass pathway for telomere maintenance in Saccharomyces cerevisiae, Mol Cell Biol, 19 (1999) 8083-8093. 
[26] H.A. Pickett, R.R. Reddel, Molecular mechanisms of activity and derepression of alternative lengthening of telomeres, Nat Struct Mol Biol, 22 (2015) 875-880.

[27] R.L. Dilley, P. Verma, N.W. Cho, H.D. Winters, A.R. Wondisford, R.A. Greenberg, Break-induced telomere synthesis underlies alternative telomere maintenance, Nature, 539 (2016) 54-58.

[28] A. Malkova, G. Ira, Break-induced replication: functions and molecular mechanism, Curr Opin Genet Dev, 23 (2013) 271-279.

[29] F.M. Roumelioti, S.K. Sotiriou, V. Katsini, M. Chiourea, T.D. Halazonetis, S. Gagos, Alternative lengthening of human telomeres is a conservative DNA replication process with features of breakinduced replication, EMBO Rep, 17 (2016) 1731-1737.

[30] J.R. Lydeard, S. Jain, M. Yamaguchi, J.E. Haber, Break-induced replication and telomeraseindependent telomere maintenance require Pol32, Nature, 448 (2007) 820-823.

[31] J.R. Lydeard, Z. Lipkin-Moore, Y.J. Sheu, B. Stillman, P.M. Burgers, J.E. Haber, Break-induced replication requires all essential DNA replication factors except those specific for pre-RC assembly, Genes Dev, 24 (2010) 1133-1144.

[32] E.V. Linardopoulou, E.M. Williams, Y. Fan, C. Friedman, J.M. Young, B.J. Trask, Human subtelomeres are hot spots of interchromosomal recombination and segmental duplication, Nature, 437 (2005) 94-100.

[33] B.B. De Vries, R. Winter, A. Schinzel, C. van Ravenswaaij-Arts, Telomeres: a diagnosis at the end of the chromosomes, J Med Genet, 40 (2003) 385-398.

[34] S. Tashiro, Y. Nishihara, K. Kugou, K. Ohta, J. Kanoh, Subtelomeres constitute a safeguard for gene expression and chromosome homeostasis, Nucleic Acids Res, 45 (2017) 10333-10349.

[35] E.J. Louis, J.E. Haber, The structure and evolution of subtelomeric $Y^{\prime}$ repeats in Saccharomyces cerevisiae, Genetics, 131 (1992) 559-574.

[36] M. Yamada, N. Hayatsu, A. Matsuura, F. Ishikawa, Y'-Help1, a DNA helicase encoded by the yeast subtelomeric $Y^{\prime}$ element, is induced in survivors defective for telomerase, J Biol Chem, 273 (1998) 33360-33366.

[37] N. Grandin, S.I. Reed, M. Charbonneau, Stn1, a new Saccharomyces cerevisiae protein, is implicated in telomere size regulation in association with Cdc13, Genes Dev, 11 (1997) 512-527.

[38] N. Grandin, M. Charbonneau, Telomerase- and Rad52-independent immortalization of budding yeast by an inherited-long-telomere pathway of telomeric repeat amplification, Mol Cell Biol, 29 (2009) 965-985.

[39] E.J. Louis, R.H. Borts, A complete set of marked telomeres in Saccharomyces cerevisiae for physical mapping and cloning, Genetics, 139 (1995) 125-136.

[40] H. Horowitz, P. Thorburn, J.E. Haber, Rearrangements of highly polymorphic regions near telomeres of Saccharomyces cerevisiae, Mol Cell Biol, 4 (1984) 2509-2517.

[41] R.J. Wellinger, V.A. Zakian, Everything you ever wanted to know about Saccharomyces cerevisiae telomeres: beginning to end, Genetics, 191 (2012) 1073-1105.

[42] S. Makovets, T.L. Williams, E.H. Blackburn, The telotype defines the telomere state in Saccharomyces cerevisiae and is inherited as a dominant non-Mendelian characteristic in cells lacking telomerase, Genetics, 178 (2008) 245-257.

[43] E.P. Mimitou, L.S. Symington, DNA end resection: many nucleases make light work, DNA Repair (Amst), 8 (2009) 983-995.

[44] L.S. Symington, Mechanism and regulation of DNA end resection in eukaryotes, Crit Rev Biochem Mol Biol, 51 (2016) 195-212.

[45] Z. Yan, C. Xue, S. Kumar, J.B. Crickard, Y. Yu, W. Wang, N. Pham, Y. Li, H. Niu, P. Sung, E.C. Greene, G. Ira, Rad52 Restrains Resection at DNA Double-Strand Break Ends in Yeast, Mol Cell, 76 (2019) 699-711 e696.

[46] C. Claussin, M. Chang, The many facets of homologous recombination at telomeres, Microb Cell, 2 (2015) 308-321.

[47] N. Grandin, C. Damon, M. Charbonneau, Cdc13 prevents telomere uncapping and Rad50dependent homologous recombination, EMBO J, 20 (2001) 6127-6139. 
[48] B. Garvik, M. Carson, L. Hartwell, Single-stranded DNA arising at telomeres in cdc13 mutants may constitute a specific signal for the RAD9 checkpoint, Mol Cell Biol, 15 (1995) 6128-6138.

[49] M. Larrivee, R.J. Wellinger, Telomerase- and capping-independent yeast survivors with alternate telomere states, Nat Cell Biol, 8 (2006) 741-747.

[50] M.K. Zubko, D. Lydall, Linear chromosome maintenance in the absence of essential telomerecapping proteins, Nat Cell Biol, 8 (2006) 734-740.

[51] D. Jain, A.K. Hebden, T.M. Nakamura, K.M. Miller, J.P. Cooper, HAATI survivors replace canonical telomeres with blocks of generic heterochromatin, Nature, 467 (2010) 223-227.

[52] O. Calvo, N. Grandin, A. Jordan-Pla, E. Minambres, N. Gonzalez-Polo, J.E. Perez-Ortin, M. Charbonneau, The telomeric Cdc13-Stn1-Ten1 complex regulates RNA polymerase II transcription, Nucleic Acids Res, 47 (2019) 6250-6268.

[53] M. Lisby, T. Teixeira, E. Gilson, V. Geli, The fate of irreparable DNA double-strand breaks and eroded telomeres at the nuclear periphery, Nucleus, 1 (2010) 158-161.

[54] Z. Ding, C.J. Wu, M. Jaskelioff, E. Ivanova, M. Kost-Alimova, A. Protopopov, G.C. Chu, G. Wang, X. Lu, E.S. Labrot, J. Hu, W. Wang, Y. Xiao, H. Zhang, J. Zhang, J. Zhang, B. Gan, S.R. Perry, S. Jiang, L. Li, J.W. Horner, Y.A. Wang, L. Chin, R.A. DePinho, Telomerase reactivation following telomere dysfunction yields murine prostate tumors with bone metastases, Cell, 148 (2012) 896-907.

[55] E.J. Louis, The chromosome ends of Saccharomyces cerevisiae, Yeast, 11 (1995) 1553-1573. 